\title{
Spin-to-orbital angular momentum conversion in spin Hall effect of light
}

\author{
Hailu Luo, Shuangchun Wen $*$ Weixing Shu, and Dianyuan Fan \\ Key Laboratory for Micro/Nano Opto-Electronic Devices of Ministry of Education, \\ College of Information Science and Engineering, \\ Hunan University, Changsha 410082, People's Republic of China
}

(Dated: May 30, 2018)

\begin{abstract}
From the viewpoint of classical electrodynamics, we identify the role of spin-to-orbital angular momentum conversion in spin Hall effect (SHE) of light. We introduce a distinct separation between spin and orbital angular momenta to clarify the spin-orbital interaction in conventional beam refraction. We demonstrate that the refractive index gradient can enhance or suppress the spin-to-orbital angular momentum conversion, and thus can control the SHE of light. We suggest that the metamaterial whose refractive index can be tailored arbitrarily may become a good candidate for amplifying or eliminating the SHE of light, and by properly facilitating the spin-to-orbital angular momentum conversion the SHE may be enhanced dramatically. The transverse spatial shifts governed by the spin-to-orbital angular momentum conversion, provide us a clear physical picture to clarify the role of refractive index gradient in the SHE of light. These findings provide a pathway for modulating the SHE of light and can be extrapolated to other physical systems.
\end{abstract}

PACS numbers: 42.25.-p, 42.79.-e, 41.20.Jb

Keywords: spin Hall effect of light, spin-to-orbital angular momentum conversion, refractive index gradient

\section{INTRODUCTION}

The development of spin photonics has taken an important step forwards due to the recently experimental verifications of the spin Hall effect (SHE) of light [1, 2]. The SHE is a transport phenomenon, in which an applied field on the spin particles leads to a spin-dependent shift perpendicular to the electric field $[3-5]$. The SHE of light can be regarded as a direct optical analogy in which the spin electrons and electric potential are replaced by spin photons and refractive index gradient. The SHE of light sometimes referred to as the Fedorov-Imbert effect, was predicted theoretically by Fedorov [6], and was experimentally confirmed by Imbert 7]. The theory was extended in a formulation which shows that the transverse spatial separation of the left and right circularly polarized components on oblique incidence directly from the total angular momentum conservation [8, 9]. More recently, the interesting effect has also been observed in scattering from dielectric spheres 10], on the direction making an angle with the propagation axis [1], and in silicon via free-carrier absorption [12].

The polarization-dependent transverse shift in the SHE of light is generally believed as a result of an effective spin-orbital interaction, which describes the mutual influence of the spin (polarization) and trajectory of the light beam [2]. There are two characteristics of the spin-orbit interaction of photons: The first one is the influence of the trajectory upon polarization [13, 14]; The second one is the reciprocal influence of the polarization upon the trajectory [15, 16]. These mechanisms have well been understood in gradient refractive index media 13 16]. In addition, the spin-orbital interaction in both inho-

${ }^{*}$ Electronic address: scwen@hnu.cn mogeneous anisotropic media 17 and in tightly focused beams [18] can be explained by spin-to-orbital angular momentum conversion. However, the physical picture of spin-orbit interaction in conventional beam refraction has not yet been fully examined. For example, the relation between refractive index gradient and spin-to-orbital angular momentum conversion is unclear. Whether spin-toorbital angular momentum conversion can be enhanced (or suppressed) by increasing (or decreasing) the refractive index gradient? Thus, the aim of this paper is to reveal the spin-to-orbital angular momentum conversion in the SHE of light from the viewpoint of classical electrodynamics.

The paper is organized as follows. First, we establish a three dimension beam propagation model for describing the SHE of light in conventional beam refraction. Our result shows that the refractive index gradient can enhance or suppress the transverse spatial shifts in SHE of light. We suggest that the metamaterial whose refractive index can be tailored arbitrarily is a good candidate to modulate the SHE of light. Next, we attempt to obtain a clear physical picture of spin-to-orbital angular momentum conversion in the SHE of light. Within the paraxial approximation, a distinct separation between spin and orbital angular momenta is introduced. We find that the SHE of light may be dramatically enhanced by facilitating the spin-to-orbital angular momentum conversion. Finally, we want to explore what role refractive-index gradient plays in the spin-to-orbital angular momentum conversion. We demonstrate that the refractive index gradient can enhance or suppress the spin-to-orbital angular momentum conversion, and thus can control the SHE of light. 


\section{THREE DIMENSION BEAM PROPAGATION MODEL}

To reveal the spin-to-orbital angular momentum conversion, we need to establish a three dimension beam propagation model for describing the SHE of light. Figure 11 illustrates the beam reflection and refraction in Cartesian coordinate system. The $z$ axis of the laboratory Cartesian frame $(x, y, z)$ is normal to the air-glass interface locating at $z=0$. We use the coordinate frames $\left(x_{a}, y_{a}, z_{a}\right)$ for central wave vector, where $a=i, r, t$ denotes incident, reflection, and transmission, respectively. In addition, we must introduce local Cartesian frames $\left(X_{a}, Y_{a}, Z_{a}\right)$ to describe an arbitrary wave vector. The electric field of the ath beam can be solved by employing the Fourier transformations 19]. The complex amplitude for the $a$ th beam can be conveniently expressed as

$$
\begin{aligned}
\mathbf{E}_{a}\left(x_{a}, y_{a}, z_{a}\right)= & \int d k_{a x} d k_{a y} \tilde{\mathbf{E}}_{a}\left(k_{a x}, k_{a y}\right) \\
& \times \exp \left[i\left(k_{a x} x_{a}+k_{a y} y_{a}+k_{a z} z_{a}\right)\right]
\end{aligned}
$$

where $k_{a z}=\sqrt{k_{a}^{2}-\left(k_{a x}^{2}+k_{a y}^{2}\right)}$ and $\tilde{\mathbf{E}}_{a}\left(k_{a x}, k_{a y}\right)$ is the angular spectrum. The approximate paraxial expression for the field in Eq. (11) can be obtained by the expansion of the square root of $k_{a z}$ to the first order [20], which yields

$$
\begin{aligned}
\mathbf{E}_{a}= & \exp \left(i k_{a} z_{a}\right) \int d k_{a x} d k_{a y} \tilde{\mathbf{E}}_{a}\left(k_{a x}, k_{a y}\right) \\
& \times \exp \left[i\left(k_{a x} x_{a}+k_{a y} y_{a}-\frac{k_{a x}^{2}+k_{a y}^{2}}{2 k_{a}} z_{a}\right)\right]
\end{aligned}
$$

In general, an arbitrary linear polarization can be decomposed into horizontal and vertical components. In the spin basis set, the angular spectrum can been written as:

$$
\begin{gathered}
\tilde{E}_{i}^{H}=\frac{1}{\sqrt{2}}\left(\tilde{\mathbf{E}}_{i+}+\tilde{\mathbf{E}}_{i-}\right), \\
\tilde{E}_{i}^{V}=\frac{1}{\sqrt{2}} i\left(\tilde{\mathbf{E}}_{i-}-\tilde{\mathbf{E}}_{i+}\right) .
\end{gathered}
$$

Here, $H$ and $V$ represent horizontal and vertical polarizations, respectively. The positive and negative signs denote the left and right components, respectively [21]. The monochromatic Gaussian beam can be formulated as a localized wave packet whose spectrum arbitrarily narrows, whose angular spectrum can be written as

$$
\tilde{\mathbf{E}}_{i \pm}=\left(\mathbf{e}_{i x} \pm i \mathbf{e}_{i y}\right) \frac{w_{0}}{\sqrt{2 \pi}} \exp \left[-\frac{w_{0}^{2}\left(k_{i x}^{2}+k_{i y}^{2}\right)}{4}\right],
$$

where $w_{0}$ is the beam waist. After the angular spectrum is known, we can obtain the field characteristics for the ath beam.

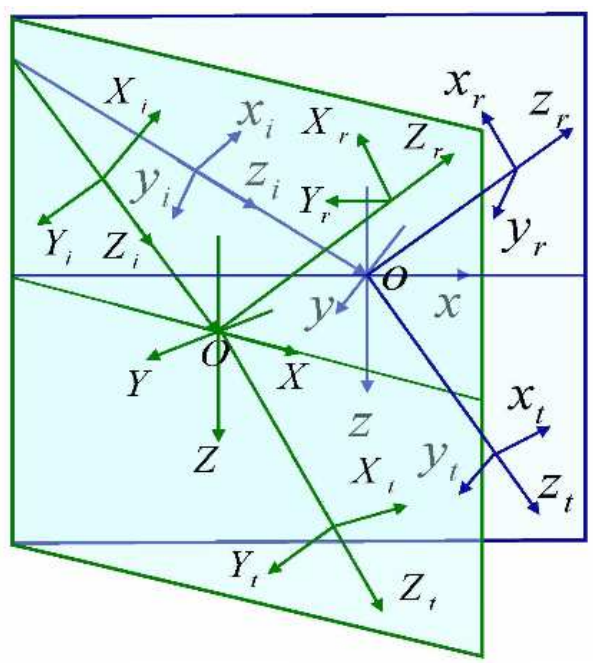

FIG. 1: (color online) Schematic illustrating the reflection and refraction of central and local wave vectors at an airglass interface in Cartesian coordinate system. $x_{a} y_{a} z_{a}$ are are reference frames for central wave vector and $X_{a} Y_{a} Z_{a}$ are local reference frames for an arbitrary wave vector. oxyz and $O X Y Z$ are the interface reference frames for central and local wave vectors, respectively.

To accurately describe the SHE of light, it is need to determine the reflection and transmission of arbitrary wave-vector components. From the central frame $x_{i} y_{i} z_{i}$ to the local frame $X_{i} Y_{i} Z_{i}$, the following three steps should be carried out. First, we transform the electric field from the reference frame $x_{i} y_{i} z_{i}$ around the $y$ axis by the incident angle $\theta_{i}$ to the frame $x y z: \tilde{E}_{x y z}=$ $m_{x_{i} y_{i} z_{i} \rightarrow x y z} \tilde{E}_{x_{i} y_{i} z_{i}}$, where

$$
m_{x_{i} y_{i} z_{i} \rightarrow x y z}=\left[\begin{array}{ccc}
\cos \theta_{i} & 0 & -\sin \theta_{i} \\
0 & 1 & 0 \\
\sin \theta_{i} & 0 & \cos \theta_{i}
\end{array}\right] .
$$

Then, we transform the electric field from the reference frame $x y z$ around the $y$ axis by an angle $k_{i y} / k_{0} \sin \theta_{i}$ to the frame $X Y Z$, and the correspondingly matrix is given by

$$
m_{x y z \rightarrow X Y Z}=\left[\begin{array}{ccc}
1 & \frac{k_{i y}}{k_{0} \sin \theta_{i}} & 0 \\
-\frac{k_{i y}}{k_{0} \sin \theta_{i}} & 1 & 0 \\
0 & 0 & 1
\end{array}\right] .
$$

Finally, we transform the electric field from the reference frame $X Y Z$ around the $y$ axis by an angle $-\theta_{i}$ to the frame $X_{i} Y_{i} Z_{i}$, and the rotation matrix can be written as

$$
m_{X Y Z \rightarrow X_{i} Y_{i} Z_{i}}=\left[\begin{array}{ccc}
\cos \theta_{i} & 0 & \sin \theta_{i} \\
0 & 1 & 0 \\
-\sin \theta_{i} & 0 & \cos \theta_{i}
\end{array}\right] .
$$

The rotation matrix of the reference frame $x_{i} y_{i} z_{i}$ around the $y$ axis by an angle $M_{x_{i} y_{i} z_{i} \rightarrow X_{i} Y_{i} Z_{i}}=$ $m_{X Y Z \rightarrow X_{i} Y_{i} Z_{i}} m_{x y z \rightarrow X Y Z} m_{x_{i} y_{i} z_{i} \rightarrow x y z}$, and we have

$$
M_{x_{i} y_{i} z_{i} \rightarrow X_{i} Y_{i} Z_{i}}=\left[\begin{array}{cc}
1 & \frac{k_{r y} \cot \theta_{i}}{k_{0}} \\
-\frac{k_{r y} \cot \theta_{i}}{k_{0}} & 1
\end{array}\right] .
$$


For an arbitrary wave vector, the reflected field is determined by $\tilde{E}_{X_{r} Y_{r} Z_{r}}=r_{p, s} \tilde{E}_{X_{i} Y_{i} Z_{i}}$, where $r_{p}$ and $r_{s}$ are the Fresnel reflection coefficients. The reflected field should be transformed from $X_{r} Y_{r} Z_{r}$ to $x_{r} y_{r} z_{r}$. Following the similar procedure, the reflected field can be obtained by carrying out three steps of transformation: $\tilde{E}_{x_{r} y_{r} z_{r}}=m_{X_{r} Y_{r} Z_{r} \rightarrow x_{r} y_{r} z_{r}} \tilde{E}_{X_{r} Y_{r} Z_{r}}$ where

$$
M_{X_{r} Y_{r} Z_{r} \rightarrow x_{r} y_{r} z_{r}}=\left[\begin{array}{cc}
1 & \frac{k_{r y} \cot \theta_{i}}{k_{0}} \\
-\frac{k_{r y} \cot \theta_{i}}{k_{0}} & 1
\end{array}\right] .
$$

Here, only the two dimension rotation matrices is taken into account, since the longitudinal component of electric field can be obtained from the divergence equation $\tilde{E}_{a z} k_{a z}=-\left(\tilde{E}_{a x} k_{a x}+\tilde{E}_{a y} k_{a y}\right)$. The reflection matrix can be written as

$$
M_{R}=M_{X_{r} Y_{r} Z_{r} \rightarrow x_{r} y_{r} z_{r}}\left[\begin{array}{cc}
r_{p} & 0 \\
0 & r_{s}
\end{array}\right] M_{x_{i} y_{i} z_{i} \rightarrow X_{i} Y_{i} Z_{i}} .
$$

The reflected angular spectrum is related to the boundary distribution of the electric field by means of the relation $\tilde{E}_{r}\left(k_{r x}, k_{r y}\right)=M_{R} \tilde{E}_{i}\left(k_{i x}, k_{i y}\right)$, and we have

$$
\left[\begin{array}{c}
\tilde{E}_{r}^{H} \\
\tilde{E}_{r}^{V}
\end{array}\right]=\left[\begin{array}{cc}
r_{p} & \frac{k_{r y}\left(r_{p}+r_{s}\right) \cot \theta_{i}}{k_{0}} \\
-\frac{k_{r y}\left(r_{p}+r_{s}\right) \cot \theta_{i}}{k_{0}} & r_{s}
\end{array}\right]\left[\begin{array}{c}
\tilde{E}_{i}^{H} \\
\tilde{E}_{i}^{V}
\end{array}\right](,
$$

It is well known that $r_{p}$ and $r_{s}$ can be expanded as a power series that can be truncated to an appropriate order $N$. By making use of Taylor series expansion based on the arbitrary angular spectrum component, $r_{p}$ and $r_{s}$ can be expanded as a polynomial of $k_{i x}$ :

$$
\begin{aligned}
r_{p, s}\left(k_{i x}\right)= & r_{p, s}\left(k_{i x}=0\right)+k_{i x}\left[\frac{\partial r_{p, s}\left(k_{i x}\right)}{\partial k_{i x}}\right]_{k_{i x}=0} \\
& +\sum_{j=2}^{N} \frac{k_{i x}^{N}}{j !}\left[\frac{\partial^{j} r_{p, s}\left(k_{i x}\right)}{\partial k_{i x}^{j}}\right]_{k_{i x}=0} .
\end{aligned}
$$

Our analysis is confined to the zero order to obtain a sufficiently good approximation. From the boundary condition, we obtain $k_{r x}=-k_{i x}$ and $k_{r y}=k_{i y}$. In fact, after the incident angular spectrum is known, Eq. (2) together with Eqs. (5) and (12) provides the paraxial expression of the reflected field:

$$
\begin{aligned}
\mathbf{E}_{r \pm}^{H}= & \frac{r_{p}}{\sqrt{\pi} w_{0}} \frac{z_{R}}{z_{R}+i z_{r}} \exp \left(i k_{r} z_{r}\right) \\
& \times \exp \left[-\frac{k_{0}}{2} \frac{x_{r}^{2}+\left(y_{r} \pm \delta_{r}^{H}\right)^{2}}{z_{R}+i z_{r}}\right] . \\
\mathbf{E}_{r \pm}^{V}= & \frac{ \pm i r_{s}}{\sqrt{\pi} w_{0}} \frac{z_{R}}{z_{R}+i z_{r}} \exp \left(i k_{r} z_{r}\right) \\
& \times \exp \left[-\frac{k_{0}}{2} \frac{x_{r}^{2}+\left(y_{r} \pm \delta_{r}^{V}\right)^{2}}{z_{R}+i z_{r}}\right] .
\end{aligned}
$$

where $\delta_{r}^{H}=\left(1+r_{s} / r_{p}\right) \cot \theta_{i} / k$ and $\delta_{r}^{V}=(1+$ $\left.r_{s} / r_{p}\right) \cot \theta_{i} / k$. Note that the above expression of reflected field coincides with the early results [22 25] with different methods.
We proceed to consider the transmitted field. Following the similar procedure, we obtain the transform matrix from $X_{t} Y_{t} Z_{t}$ to $x_{t} y_{t} z_{t}$ as

$$
M_{X_{t} Y_{t} Z_{t} \rightarrow x_{t} y_{t} z_{t}}=\left[\begin{array}{cc}
1 & \frac{k_{t y} \cos \theta_{t}}{k_{0} \sin \theta_{i}} \\
-\frac{k_{t y} \cos \theta_{t}}{k_{0} \sin \theta_{i}} & 1
\end{array}\right],
$$

where $\theta_{t}$ is the transmitted angle. For an arbitrary wave vector, the transmitted field is determined by $\tilde{E}_{X_{t} Y_{t} Z_{t}}=$ $t_{p, s} \tilde{E}_{X_{i} Y_{i} Z_{i}}$, where $t_{p}$ and $t_{s}$ are the Fresnel transmission coefficients. Hence, the transmitted field should be transformed from $X_{r} Y_{r} Z_{r}$ to $x_{t} y_{t} z_{t}$, and the transmission matrix can be written as

$$
M_{T}=M_{X_{t} Y_{t} Z_{t} \rightarrow x_{t} y_{t} z_{t}}\left[\begin{array}{cc}
t_{p} & 0 \\
0 & t_{s}
\end{array}\right] M_{x_{i} y_{i} z_{i} \rightarrow X_{i} Y_{i} Z_{i}} .
$$

The transmitted angular spectrum is related to the boundary distribution of the electric field by means of the relation $\tilde{E}_{t}\left(k_{t x}, k_{t y}\right)=M_{T} \tilde{E}_{i}\left(k_{i x}, k_{i y}\right)$, and can be written as

$$
\left[\begin{array}{c}
\tilde{E}_{t}^{H} \\
\tilde{E}_{t}^{V}
\end{array}\right]=\left[\begin{array}{cc}
t_{p} & \frac{k_{t y}\left(t_{p}-\eta t_{s}\right) \cot \theta_{i}}{k_{0}} \\
\frac{k_{t y}\left(\eta t_{p}-t_{s}\right) \cot \theta_{i}}{k_{0}} & t_{s}
\end{array}\right]\left[\begin{array}{c}
\tilde{E}_{i}^{H} \\
\tilde{E}_{i}^{V}
\end{array}\right]
$$

where $\eta=\cos \theta_{t} / \cos \theta_{i}$. Based on the Taylor series expansion, the Fresnel transmission coefficients $t_{p}$ and $t_{s}$ can be written as

$$
\begin{aligned}
t_{p, s}\left(k_{i x}\right)= & t_{p, s}\left(k_{i x}=0\right)+k_{i x}\left[\frac{\partial t_{p, s}\left(k_{i x}\right)}{\partial k_{x}}\right]_{k_{i x}=0} \\
& +\sum_{j=2}^{N} \frac{k_{i x}^{N}}{j !}\left[\frac{\partial^{j} t_{p, s}\left(k_{i x}\right)}{\partial k_{i x}^{j}}\right]_{k_{i x}=0} .
\end{aligned}
$$

Note that the Fresnel coefficients are real in the regime of partial reflection and transmission, $\sin \theta_{i}<n$. From the Snell's law under the paraxial approximation, we obtain $k_{t x}=k_{i x} / \eta$ and $k_{t y}=k_{i y}$. Substituting Eqs. (5) and (18) into Eq. (2), we obtain the transmitted field:

$$
\begin{aligned}
\mathbf{E}_{t \pm}^{H}= & \frac{t_{p}}{\sqrt{\pi} w_{0}} \frac{z_{R y} \exp \left(i k_{t} z_{t}\right)}{\sqrt{\left(z_{R x}+i z_{t}\right)\left(z_{R y}+i z_{t}\right)}} \\
& \times \exp \left[-\frac{n k_{0}}{2}\left(\frac{x_{t}^{2}}{z_{R x}+i z_{t}}+\frac{\left(y_{t} \pm \delta_{t}^{H}\right)^{2}}{z_{R y}+i z_{t}}\right)\right] \\
\mathbf{E}_{t \pm}^{V}= & \frac{ \pm i t_{s}}{\sqrt{\pi} w_{0}} \frac{z_{R y} \exp \left(i k_{t} z_{t}\right)}{\sqrt{\left(z_{R x}+i z_{t}\right)\left(z_{R y}+i z_{t}\right)}} \\
& \times \exp \left[-\frac{n k_{0}}{2}\left(\frac{x_{t}^{2}}{z_{R x}+i z_{t}}+\frac{\left(y_{t} \pm \delta_{t}^{V}\right)^{2}}{z_{R y}+i z_{t}}\right)\right]
\end{aligned}
$$

Here, $\delta_{t}^{H}=\left(1-\eta t_{s} / t_{p}\right) \cot \theta_{i} / k$ and $\delta_{t}^{V}=(1-$ $\left.\eta t_{p} / t_{s}\right) \cot \theta_{i} / k$. The interesting point we want to stress is that there are two different Rayleigh lengths, $z_{R x}=$ $n \eta^{2} k_{0} w_{0}^{2} / 2$ and $z_{R y}=n k_{0} w_{0}^{2} / 2$, characterizing the spreading of the beam in the direction of $x$ and $y$ axes, respectively. Up to now, we have established a general propagation model to describe the reflected and transmitted fields. 

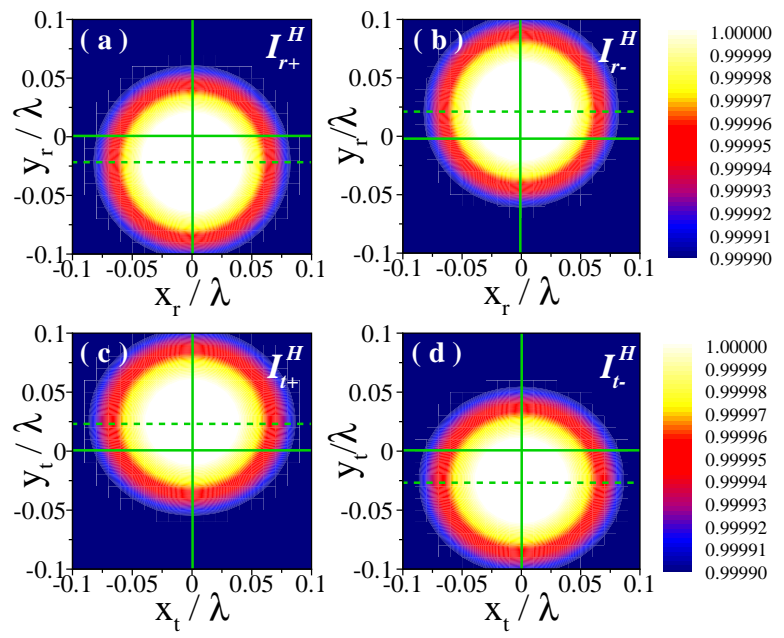

FIG. 2: (color online) The SHE of light manifests itself as polarization-dependent transverse shifts of field centroid. [(a), (b)] Intensity distribution of the reflected field for left and right circularly polarized components, respectively. [(c), (d)] Intensity distribution of the transmitted field for left and right circularly polarized components, respectively. The refractive index of the glass is $n=1.515$ and the incident angle is chosen as $\theta_{i}=\pi / 6$. The beam waist is chosen as $w_{0}=10 \lambda$. The intensity distributions in the plane $z_{a}=0$ are plotted in normalized units.

\section{ROLE OF THE REFRACTIVE INDEX GRADIENT}

It is well known that the SHE of light manifests itself as polarization-dependent transverse shifts in the process of reflection and refraction. To reveal the SHE of light, we now determine the transverse spatial shifts of field centroid. The time-averaged linear momentum density associated with the electromagnetic field can be shown to be 26]

$$
\mathbf{p}_{a}(\mathbf{r})=\frac{1}{2 c^{2}} \operatorname{Re}\left[\mathbf{E}_{a}(\mathbf{r}) \times \mathbf{H}_{a}^{*}(\mathbf{r})\right]
$$

where the magnetic field can be obtained by $\mathbf{H}_{a}=$ $-i k_{a}^{-1} \nabla \times \mathbf{E}_{a}$. The intensity distribution of electromagnetic fields is closely linked to the longitudinal momentum currents $I\left(x_{a}, y_{a}, z_{a}\right) \propto \mathbf{p}_{a} \cdot \mathbf{e}_{a z}$.

Figure 2 shows the transverse spatial shifts of beam centroid in an air-glass interface. In the case of reflection, the left circularly polarized component exhibits a negative shift [Fig. 2(a)]. For the right circular polarization, however, presents a positive shift [Fig. 2(b)]. In the case of transmission, the left circularly polarized component exhibits a positive transverse shift [Fig. 2(c)]. For the right circularly polarized component, however, presents a negative transverse shift [Fig. 2(d)]. This interesting phenomenon gives a clear evidence of polarization-dependent splitting of field intensity in the SHE of light. The transverse shifts are polarization-dependent, and thus can be regarded as the influence of the polarization upon trajectory. In the air-glass interface, the transverse shifts are just a few tens of nanometers, which can be observed via weak measurements [1, 27]. However, how to amplify this tiny effect is still an open problem.

As we know that the refractive index gradient acts as the electric potential gradient in the electronic systems. Now a question naturally arises: Whether can the refractive index gradient enhance the transverse shift in the SHE of light? To answer this question we need to obtain a relation between the transverse shift and the refractive index gradient. At any given plane $z_{a}=$ const., the transverse spatial shift of beam centroid compared to the geometrical-optics prediction is given by

$$
\Delta y_{a}=\frac{\iint y_{a} I\left(x_{a}, y_{a}, z_{a}\right) \mathrm{d} x_{a} \mathrm{~d} y_{a}}{\iint I\left(x_{a}, y_{a}, z_{a}\right) \mathrm{d} x_{a} \mathrm{~d} y_{a}} .
$$

Note that the transverse spatial shift is $z_{a}$-independent, while the transverse angular shift can be regarded as a small shift inclining from the $z_{a}$ axis. In addition, the field also experience a longitudinal spatial shift [28 30 . and a longitudinal angular shift [31, 32]. Note that the angle shift means that the Snell's law cannot accurately describe the beam refraction phenomenon 33].

We first consider the transverse spatial shift of the reflected field. After substituting the reflected field Eqs. (14) and (15) into Eq. (23), we obtain the transverse spatial shifts as

$$
\begin{aligned}
& \Delta y_{r \pm}^{H}=\mp \frac{\lambda}{2 \pi}\left(1+r_{s} / r_{p}\right) \cot \theta_{i} \\
& \Delta y_{r \pm}^{V}=\mp \frac{\lambda}{2 \pi}\left(1+r_{p} / r_{s}\right) \cot \theta_{i} .
\end{aligned}
$$

For an arbitrary linearly polarized beam, the transverse spatial shift of the reflected field is given by

$$
\Delta y_{r \pm}=\cos \gamma_{r}^{2} \Delta y_{r \pm}^{H}+\sin \gamma_{r}^{2} \Delta y_{r \pm}^{V}
$$

where $\gamma_{r}$ is the reflected polarization angle. In the frame of classical electrodynamics, the reflection polarization angle is determined by:

$$
\begin{aligned}
& \cos \gamma_{r}=\frac{\cos \gamma_{i} r_{p}}{\sqrt{\cos ^{2} \gamma_{i}^{2} r_{p}^{2}+\sin ^{2} \gamma_{i}^{2} r_{s}^{2}}}, \\
& \sin \gamma_{r}=\frac{\sin \gamma_{i} r_{s}}{\sqrt{\cos ^{2} \gamma_{i}^{2} r_{p}^{2}+\sin ^{2} \gamma_{i}^{2} r_{s}^{2}}} .
\end{aligned}
$$

Here, $\gamma_{i}$ is the incident polarization angle. Under the limit of ultra-high refractive index gradient, the transverse spatial shifts can be written as $\Delta y_{r \pm}=0$. This simple result means that the recent advent of a new class of metamaterial with ultra-high refractive index [34] is a possible candidate to eliminate the transverse spatial shift in the reflected field.

We next consider the transverse spatial shifts of the transmitted field. After substituting the transmitted field Eqs. (20) and (21) into Eq. (23), we have

$$
\Delta y_{t \pm}^{H}=\mp \frac{\lambda}{2 \pi}\left(1-\eta t_{s} / t_{p}\right) \cot \theta_{i}
$$




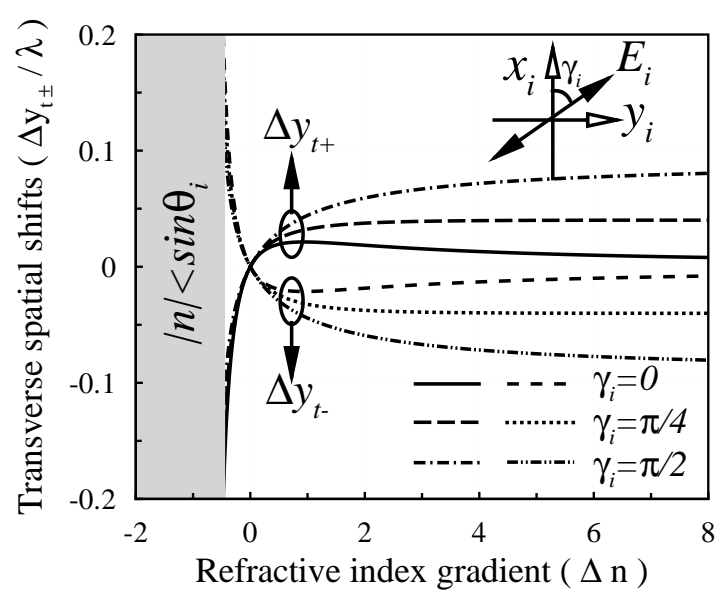

FIG. 3: (a) Schematically shows the SHE of light manifests itself as spin-dependent splitting. The transverse spatial shifts of transmitted field $\Delta y_{t \pm} / \lambda$ versus the thickness of air gap $d_{2}$. The incident polarization angles are chooser as (b) $\theta_{i}=0$, (c) $\theta_{i}=\pi / 4$, and $\theta_{i}=\pi / 2$. Other parameters are chosen to be the same as in Fig. 2 .

$$
\Delta y_{t \pm}^{V}=\mp \frac{\lambda}{2 \pi}\left(1-\eta t_{p} / t_{s}\right) \cot \theta_{i}
$$

For an arbitrary linearly polarized wave-packet, the transverse spatial shifts of the transmitted field are given by

$$
\Delta y_{t \pm}=\cos \gamma_{t}^{2} \Delta y_{t \pm}^{H}+\sin \gamma_{t}^{2} \Delta y_{t \pm}^{V},
$$

where the transmission polarization angle $\gamma_{t}$ is determined by

$$
\begin{aligned}
& \cos \gamma_{t}=\frac{\cos \gamma_{i} t_{p}}{\sqrt{\cos ^{2} \gamma_{i}^{2} t_{p}^{2}+\sin ^{2} \gamma_{i}^{2} t_{s}^{2}}} \\
& \sin \gamma_{t}=\frac{\sin \gamma_{i} t_{s}}{\sqrt{\cos ^{2} \gamma_{i}^{2} t_{p}^{2}+\sin ^{2} \gamma_{i}^{2} t_{s}^{2}}}
\end{aligned}
$$

Note that the above expression coincides well with the early results [1] with the quantum method. Our scheme shows that the SHE of light can be explained from the classic electrodynamics. Under the limit of ultra-high refractive index gradient, the transmitted field tends to reach a saturation value:

$$
\Delta y_{t \pm}= \pm \frac{\lambda \sin \theta_{i} \cos ^{2} \gamma_{i}^{2}}{2 \pi\left(\cos ^{2} \gamma_{i}^{2}+\cos ^{2} \theta_{i}^{2} \sin ^{2} \gamma_{i}^{2}\right)}
$$

Up to now, we have described the polarization-dependent splitting in the SHE of light from the viewpoint of pure classical electrodynamics.

We proceed to examine the role of refractive index gradient (i.e., $\Delta n=|n|-1$ ) in the SHE of light. The normalized transverse spatial shifts of transmitted field for various refractive index gradients as shown in Fig. 3. We first consider the wave packet incident from air to a

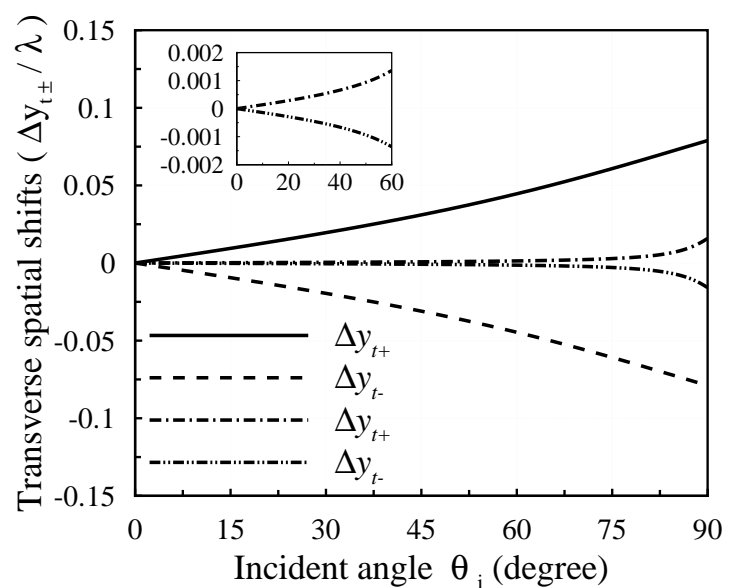

FIG. 4: The normalized transverse spatial shifts of transmitted field $\Delta y_{t \pm} / \lambda$ versus incident angles $\theta_{i}$ in the metamaterial with $\varepsilon=-1.00+0.028 i$ and $\mu=-1.01+0.028 i$ (dashed-dotted and dashed-doted-dotted lines). As a comparison, the conventional medium is chosen as the glass with $n=+1.515$ (solid and dashed lines). The incident polarization angle choose as $\gamma_{i}=0$. Other parameters are chosen to be the same as in Fig. 2 Inset: Zoomed-in view of the transverse spatial shifts at the air-metamaterial interface.

low-refractive-index medium $(\Delta n<0)$. For the left circularly polarized component, the field centroid exhibits a negative transverse shift. For the right circularly polarized component, the beam centroid also presents a transverse spatial shift, but in an opposite direction. We next consider the wave packet incident from air to a highrefractive-index medium $(\Delta n>0)$. For a certain polarized component, we find that the SHE of light is reversed when the refractive index gradient is inverted. It is clearly shown that the transverse spatial shifts increases with the increase of the refractive index gradient $\Delta n$. Within the regime of low refractive index, the transverse spatial shifts are enhanced sharply with the increase of $\Delta n$. When the refractive index $|n|<\sin \theta_{i}$, the beam is totally reflected. While in the regime of high refractive index, the transverse spatial shifts tend to reach a saturation value. In addition, the transverse spatial shifts are also sensitive to the incident polarization angle as clearly shown in the figure. Hence, we can enhance or suppress the transverse spatial shifts in SHE of light by modulating the refractive index gradient and incident polarization angle.

How to attenuate the SHE of light is not of pure academic interest, owing to the requirement of eliminating the transverse shifts, such as reflection, refraction, and focusing in optical experiments. We suggest that the metamaterial whose refractive index can be tailored arbitrarily [35, 36] may become a good candidate. In order to accurately describe how the metamaterial attenuate the SHE of light, it is necessary to include material dispersion and losses. Thus, a certain dispersion relation, such as the Lorentz medium model, should be introduced. 
The relative constitutive parameters are

$$
\begin{aligned}
\varepsilon(\omega) & =1-\frac{\omega_{e p}^{2}}{\omega^{2}-\omega_{e o}^{2}+i \Gamma_{e} \omega}, \\
\mu(\omega) & =1-\frac{F \omega_{m p}^{2}}{\omega^{2}-\omega_{m o}^{2}+i \Gamma_{m} \omega} .
\end{aligned}
$$

To avoid the trouble involving in a certain value of frequency, we assume the material parameters are $\omega_{e o}=$ $\omega_{m o}=\omega_{o}, \omega_{e p}^{2}=\omega_{m p}^{2}=2 \omega_{o}^{2}, F=1.005$, and $\Gamma_{e}=\Gamma_{m}=$ $0.01 \omega_{0}$. Figure 4 shows the normalized transverse spatial shifts of transmitted field versus incident angle at the airmetamaterial interface. For the comparison, we also plot the transverse spatial shifts at the air-glass interface. In a large range of incident angles, the metamaterial is a good candidate for suppressing the SHE of light in the process of refraction. In the ideal case $(\varepsilon=-1$ and $\mu=-1)$, the transverse shift can be eliminated completely. This is another reason why a simple planar slab provides us with a perfect lens without aberration [37]. As we will see in the following, the spin-to-orbital angular momentum conversion can be used to explain the inherent physics underlying these intriguing phenomena.

\section{SPIN-TO-ORBITAL ANGULAR MOMENTUM CONVERSION}

It should be noted is that the spin-orbital interaction in both inhomogeneous anisotropic media [17] and in tightly focused beams [18] can be explained by spin-to-orbital angular momentum conversion. To obtain a clear physical picture of the SHE of light, we introduce a distinct separation between spin and orbital angular momenta to clarify the spin-orbital interaction. The momentum current can be regarded as the combined contributions of spin and orbital parts:

$$
\mathbf{p}_{a}=\mathbf{p}_{a}^{O}+\mathbf{p}_{a}^{S} .
$$

Here, the orbital term is determined by the macroscopic energy current with respect to an arbitrary reference point and does not depend on the polarization. The spin term, on the other hand, relates to the phase between orthogonal field components and is completely determined by the state of polarization [38]. In a monochromatic optical beam, the spin and orbital currents can be respectively written in the form:

$$
\begin{aligned}
& \mathbf{p}_{a}^{O}=\operatorname{Im}\left[\mathbf{E}_{a}^{*} \cdot(\nabla) \mathbf{E}_{a}\right], \\
& \mathbf{p}_{a}^{S}=\operatorname{Im}\left[\left(\mathbf{E}_{a} \cdot \nabla\right) \mathbf{E}_{a}^{*}\right],
\end{aligned}
$$

where $\mathbf{E}_{a}^{*} \cdot(\nabla) \mathbf{E}_{a}=E_{a x}^{*} \nabla E_{a x}+E_{a y}^{*} \nabla E_{a y}+E_{a z}^{*} \nabla E_{a z}$ is the invariant Berry notation [39]. It has been shown that both spin and orbital currents originate from the beam transverse inhomogeneity and their components are directly related to the azimuthal and radial derivatives of the beam profile parameters. The orbital currents are
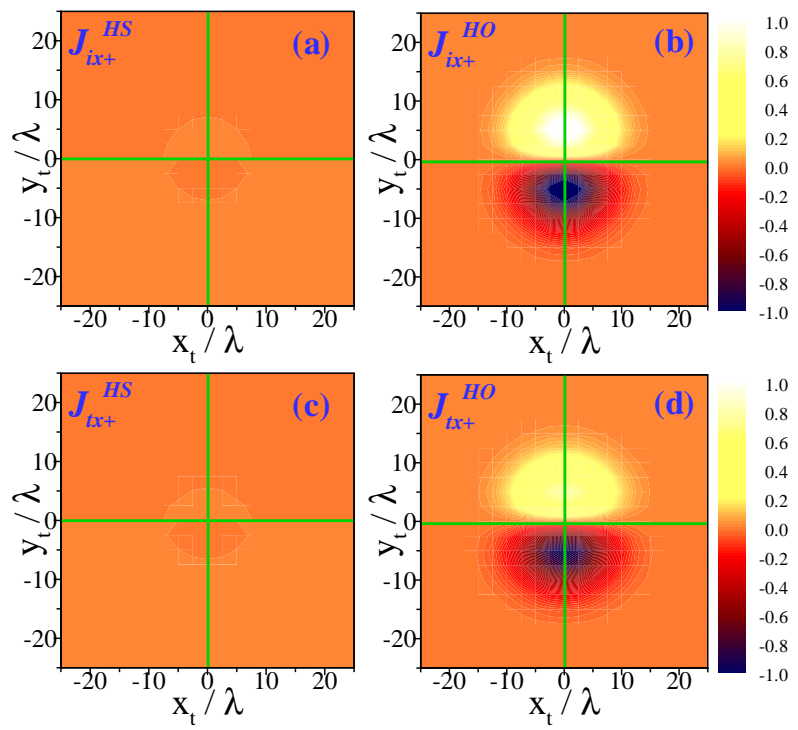

FIG. 5: (color online) The transverse angular momentum density of left circularly polarized component. [(a) and (b)] Incident fields: $j_{i x+}^{H S}$ and $j_{i x+}^{H O}$. [(c) and (d)] Transmitted fields: $j_{t x+}^{H S}$ and $j_{t x+}^{H O}$. The cross section is chosen as $z_{t}=0$ and the intensity is plotted in normalized units. Other parameters are the same as those in Fig. 2 .

mainly produced by the phase gradient, while the spin currents are orthogonal to the intensity gradient.

We proceed to analysis the angular momentum density for each of individual wave packet, which can be written as

$$
\mathbf{j}_{a}(\mathbf{r})=\mathbf{r}_{a} \times \mathbf{p}_{a}\left(\mathbf{r}_{a}\right) .
$$

Within the paraxial approximation, the angular momentum can be divided into the orbital and spin angular parts: $\mathbf{j}_{a}=\mathbf{j}_{a}^{O}+\mathbf{j}_{a}^{S}$ [40, 41], it follows that

$$
\begin{aligned}
& \mathbf{j}_{a}^{O}=\mathbf{r}_{a} \times \mathbf{p}_{a}^{O}, \\
& \mathbf{j}_{a}^{S}=\mathbf{r}_{a} \times \mathbf{p}_{a}^{S} .
\end{aligned}
$$

It should be mentioned that this separation still hold beyond the paraxial approximation [42]. The transverse angular momentum density $j_{x}$ can be regarded as the combined contributions of orbital and spin parts:

$$
\begin{aligned}
& j_{a x}^{O}=y_{a} p_{a z}^{O}-z_{a} p_{a y}^{O}, \\
& j_{a x}^{S}=y_{a} p_{a z}^{S}-z_{a} p_{a y}^{S} .
\end{aligned}
$$

The time-averaged linear and angular momenta can be obtained by integrating over the whole $x-y$ plane [26]

$$
\begin{aligned}
J_{a} & =\iint j_{a} \mathrm{~d} x \mathrm{~d} y, \\
P_{a} & =\iint p_{a} \mathrm{~d} x \mathrm{~d} y .
\end{aligned}
$$


The transverse orbital angular momentum would appear if the spin-to-orbital angular momentum conversion occurs in the process of reflection and refraction. It should be mentioned that the transverse orbital angular momentum can lead to a transverse shift of field centroid even in free space [43]. In the plane $z_{a}=0$, after substituting Eqs (43) and (44) into Eqs (45) and (46) we obtain

$$
\Delta y_{a}=\frac{J_{a x}^{S}}{P_{a z}^{S}}=\frac{J_{a x}^{O}}{P_{a z}^{O}} .
$$

Figure 5 plots the transverse spin and orbital momentum currents for incident and transmitted fields. It is clearly shows that the transverse orbital angular momentum plays a dominant role in the SHE of light. Compare Fig 5(b) with (d), however, it is not very clear why the transmitted field exhibits the SHE of light. Thus, it is necessary to determine the transverse orbital angular momentum. From Eq. (45), we can obtain $j_{i x}^{O}=0$ and $J_{t x}^{O} \neq 0$. The presence of transverse orbital angular momentum $J_{t x}^{O}$ provide a direct evidence for the spin-toorbital angular momentum conversion. In the $\mathrm{SHE}$ of light associated with refraction [1], reflection [44], and tunneling [45], the transverse spatial shifts are sensitive to the incident polarization angles.

We proceed to explore the role of refractive index gradient in spin-to-orbital angular momentum conversion. The $z$ component of total angular momentum $J_{a z}$ for the $a$ th beam can be represented as a sum of the extrinsic orbital angular momentum $J_{a z}^{O}$ and the intrinsic spin angular momentum $J_{a z}^{S}$, i.e., $J_{a z}=J_{a z}^{O}+J_{a z}^{S}$ [46]. The $z$ component of the orbital angular momenta are given by $J_{i z}=0, J_{r z}=-\Delta y_{r} k_{r} \sin \theta_{r}$, and $J_{t z}=-\Delta y_{t} k_{t} \sin \theta_{t}$. for incident, reflected, and transmitted wave packets, respectively. In the laboratory Cartesian frame $(x, y, z)$, the $z$ component of the orbital angular momenta are given by

$$
\begin{gathered}
J_{i z \pm}^{O}=0 \\
J_{r z \pm}^{O}= \pm \frac{\left(r_{p}^{2}+r_{s}^{2}-2 r_{p} r_{s}\right) \cos \theta_{i}}{r_{p}^{2}+r_{s}^{2}} \\
J_{z t \pm}^{O}= \pm \frac{\left(t_{p}^{2}+t_{s}^{2}-2 \eta t_{p} t_{s}\right) \cos \theta_{i}}{t_{p}^{2}+t_{s}^{2}}
\end{gathered}
$$

The $z_{a}$ components of spin angular momentum for the ath beam is respectively described by

$$
\begin{gathered}
J_{i z \pm}^{S}= \pm \cos \theta_{i} \\
J_{r z \pm}^{S}= \pm \frac{2 r_{p} r_{s}}{r_{p}^{2}+r_{s}^{2}} \cos \theta_{r} \\
J_{t z \pm}^{S}= \pm \frac{2 t_{p} t_{s}}{t_{p}^{2}+t_{s}^{2}} \cos \theta_{t}
\end{gathered}
$$

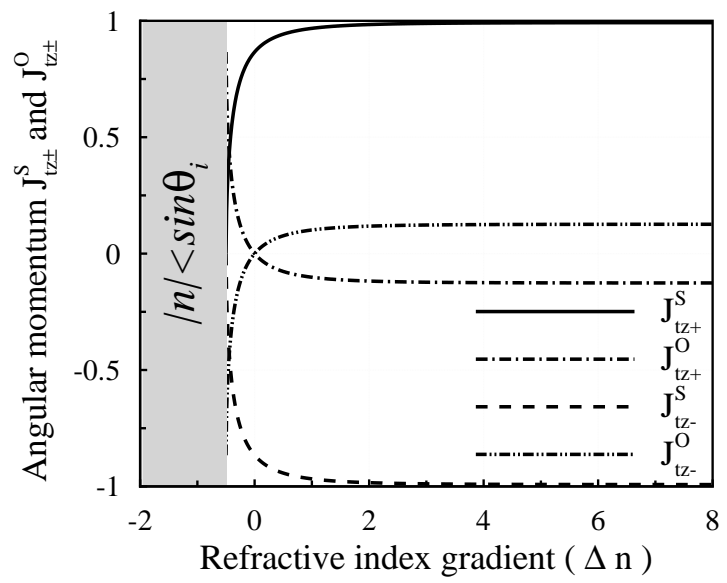

FIG. 6: The refractive index gradient $\Delta n$ induces the spinto-orbital angular momentum conversion. The incident wave packets are chosen as left circular polarization and right circular polarization. Other parameters are chosen to be the same as in Fig. 2

From Eqs. (48)-(50) and Eqs. (51)-(53), we find that the angular momenta fulfill the conservation law:

$$
Q_{r} J_{r z \pm}+Q_{t} J_{t z \pm}=J_{i z \pm}
$$

Here, $Q_{r}=\left(r_{p}^{2}+r_{s}^{2}\right) / 2$ and $Q_{t}=n \eta\left(t_{p}^{2}+t_{s}^{2}\right) / 2$ are the energy reflection and energy transmission coefficients, respectively. Note that the transverse angular shifts, which governed by the linear momentum conservation law, are not discussed in this paper.

To obtain a clear physical picture of the SHE of light, the spin-to-orbital angular momentum conversion induced by refractive index gradient is depicted in Fig. 6. For the left circularly polarized component, the spin angular momentum $J_{t z+}^{S}$ monotonously increase while the orbital angular momentum $J_{t z+}^{O}$ monotonously decreases with the increase of the refractive index gradient $\Delta n$. For the right circular polarization, both the spin angular momentum $J_{t z-}^{S}$ and the orbital one $J_{t z-}^{O}$ present oppositive features. When the refractive index gradient continues increasing, the spin-to-orbital angular momentum conversion appears to reach a saturation value:

$$
J_{t z \pm}^{O}=\mp \frac{\cos \theta_{i} \sin ^{2} \theta_{i}}{1+\cos ^{2} \theta_{i}} .
$$

This gives a good explanation of the intriguing phenomena why the transverse spatial shifts tends to saturation values in the Fig. 3. The metamaterial with $n=-1$ is a good candidate to eliminate the SHE of light in the refraction, since the spin-to-orbital angular momentum conversion is impressed completely. The spin-to-orbital angular momentum conversion can be enhanced in the region of low refractive index gradient as shown in Fig. 6. As a result, the transverse spatial shifts are also be amplified in this region. However, when the refractive index $|n|<\sin \theta_{i}$ the wave pack is totally reflected. By properly facilitating the spin-to-orbital angular momentum conversion the SHE may be enhanced dramatically. 
We now give a very simple way to understand how the refractive index gradient enhance the spin-to-orbital angular momentum conversion in the SHE of light. We attempt to perform analyses on the $z$ component of the total angular momentum for each of individual photons, i.e., $J_{i z}=J_{t z}$ [47, 48]. The total angular momentum conservation law for single photon is given by

$$
J_{t z \pm}^{O} \pm \cos \theta_{t}= \pm \cos \theta_{i}
$$

where $J_{t z \pm}^{O}=-\Delta y_{t \pm} k_{t} \sin \theta_{t}$. When the photons penetrate from air into a low-refractive-index medium $(\Delta n<$ $0)$, the incident angle is less than the transmitted angle $\theta_{i}<\theta_{t}$. For the left circularly polarized photons, the $z_{t}$ component of spin angular momentum $+\cos \theta_{t}$ decreases after entering the medium. Because of the conservation law, the total angular momentum must remain unchanged. To conserve the total angular momentum, the photons must move to the direction $-y\left(\Delta y_{t+}<0\right)$ and thus generate a positive orbital angular momentum $\left(J_{t z+}^{O}>0\right)$. For the right circularly polarized photons, the $z$ component of spin angular momentum $-\cos \theta_{t}$ increases. In this case, the photons must move to the direction $+y\left(\Delta y_{t-}>0\right)$ and induce a negative orbital angular momentum $\left(J_{t z-}^{O}<0\right)$. When the photons enter into a high-refractive-index medium $(\Delta n>0)$, the incident angle is larger than the transmitted angle $\theta_{i}>\theta_{t}$. As a result, the orbital angular momentum reverses its direction.

\section{CONCLUSIONS}

In conclusion, we have identified the role of spin-toorbital angular momentum conversion in SHE of light.
We have demonstrated that the refractive index gradient can enhance or suppress the spin-to-orbital angular momentum conversion, and thus can control the SHE of light. The recent advent of metamaterial whose refractive index can be tailored arbitrarily seems to be an available candidate to amplify or eliminate the SHE of light. However, the spin-to-orbital angular momentum conversion in a ultra-large refractive index gradient is limited by a saturation value. Fortunately, the SHE of light can be dramatically amplified by plasmonic nanostructure 49 52. In addition, the SHE of light can also be noticeably enhanced when the beam carries orbital angular momentum $53-56]$. Hence, the exploration of spin-to-orbital angular momentum conversion in these systems would be very interesting. The transverse spatial shifts governed by the spin-to-orbital angular momentum conversion, give us a clear physical picture to clarify the role of refractive index gradient in the SHE of light. These findings provide a pathway for modulating the SHE of light, and thereby open the possibility for developing new nano-photonic devices. Because of the close similarity in optical physics [1, 2], condensed matter [3 -5], and highenergy physics [57, 58], by properly facilitating the spinto-orbital angular momentum conversion, the SHE may be enhanced dramatically in these physical systems.

\section{Acknowledgments}

One of authors (H.L.) thanks Dr. W. Löffler for helpful discussions. This research was partially supported by the National Natural Science Foundation of China (Grants Nos. 61025024, 11074068, and 10904036).
[1] O. Hosten and P. Kwiat, Science 319, 787 (2008).

[2] K. Y. Bliokh, A. Niv, V. Kleiner, and E. Hasman, Nature Photon. 2, 748 (2008).

[3] S. Murakami, N. Nagaosa, and S. C. Zhang, Science 301, 1348 (2003).

[4] J. Sinova, D. Culcer, Q. Niu, N. A. Sinitsyn, T. Jungwirth, and A. H. MacDonald, Phys. Rev. Lett. 92, 126603 (2004).

[5] J. Wunderlich, B. Kaestner, J. Sinova, and T. Jungwirth, Phys. Rev. Lett. 94, 047204 (2005).

[6] F. I. Fedorov, Dokl. Akad. Nauk SSSR 105, 465 (1955).

[7] C. Imbert, Phys. Rev. D 5, 787 (1972).

[8] M. Onoda, S. Murakami, and N. Nagaosa, Phys. Rev. Lett. 93, 083901 (2004).

[9] K. Y. Bliokh and Y. P. Bliokh, Phys. Rev. Lett. 96, 073903 (2006).

[10] D. Haefner, S. Sukhov, and A. Dogariu, Phys. Rev. Lett. 102, 123903 (2009).

[11] A. Aiello, N. Lindlein, C. Marquardt, and G. Leuchs, Phys. Rev. Lett. 103, 100401 (2009).

[12] J.-M. Ménard, A. E. Mattacchione, H. M. van Driel, C. Hautmann, and M. Betz, Phys. Rev. B 82, 045303
(2010).

[13] R. Y. Chiao and Y. S. Wu, Phys. Rev. Lett. 57, 933 (1986).

[14] A. Tomita and R. Y. Chiao, Phys. Rev. Lett. 57, 937 (1986).

[15] A. V. Dooghin, N. D. Kundikova, V. S. Liberman, and B. Y. Zeldovich, Phys. Rev. A 45, 8204 (1992).

[16] V. S. Liberman and B. Y. Zeldovich, Phys. Rev. A 46, 5199 (1992).

[17] L. Marrucci, C. Manzo, and D. Paparo, Phys. Rev. Lett. 96, 163905 (2006).

[18] Y. Zhao, J. S. Edgar, G. D. M. Jeffries, D. McGloin, and D. T. Chiu, Phys. Rev. Lett. 99, 073901 (2007).

[19] J. W. Goodman, Introduction to Fourier Optics (McGraw-Hill, New York, 1996).

[20] M. Lax, W. H. Louisell, and W. McKnight, Phys. Rev. A 11, 1365 (1975).

[21] R. A. Beth, Phys. Rev. 50, 115 (1936).

[22] K. Y. Bliokh and Y. P. Bliokh, Phys. Rev. E 75, 066609 (2007).

[23] A. Aiello and J. P. Woerdman, Opt. Lett. 33, 1437 (2008) 
[24] C. Menzel, C. Rockstuhl, T. Paul, S. Fahr, and F. Lederer, Phys. Rev. A 77, 013810 (2008).

[25] H. Luo, S. Wen, W. Shu, Z. Tang, Y. Zou, and D. Fan, Phys. Rev. A 80, 043810 (2009).

[26] J. D. Jackson, Classical Electrodynamics (Wiley, New York, 1999).

[27] C. M. Krowne, Phys. Lett. A 373, 466 (2009).

[28] F. Goos and H. Hächen, Ann. Phys. 1, 333 (1947).

[29] M. Merano, A. Aiello, G. W. 't Hooft, M. P. van Exter, E. R. Eliel, and J. P. Woerdman, Opt. Express 15, 15928 (2007).

[30] W. Löfler, M. P. van Exter, G. W. 't Hooft, E. R. Eliel, K. Hermans, D. J. Broer, J. P. Woerdman, Opt. Commun. 283, 3367 (2010).

[31] M. Merano, A. Aiello, M. P. van Exter, and J. P. Woerdman, Nature Photon. 3, 337 (2009).

[32] A. Aiello, M. Merano, and J. P. Woerdman, Phys. Rev. A 80, 061801(R) (2009).

[33] C. Duval, Z. Horváth, and P. A. Horváthy, Phys. Rev. D 74, 021701(R) (2006).

[34] J. Shin, J. T. Shen, and S. Fan, Phys. Rev. Lett. 102, 093903 (2009).

[35] D. R. Smith, J. B. Pendry, and M. C. K. Wiltshire, Science 305, 788 (2004).

[36] J. Pendry, D. Schurig, and D. Smith, Science 312, 1780 (2006).

[37] J. B. Pendry, Phys. Rev. Lett. 85, 3966 (2000).

[38] A. Y. Bekshaev and M. S. Soskin, Opt. Commun. 271, 332 (2007).

[39] M. V. Berry, J. Opt. A: Pure Appl. Opt. 11, 094001 (2009).

[40] L. Allen, M. W. Beijersbergen, R. J. C. Spreeuw, and J. P. Woerdman, Phys. Rev. A 45, 8185 (1992).

[41] M. J. Padgett and L. Allen, Opt. Commun. 121, 36
(1995).

[42] S. M. Barnett, J. Opt. B 4, S7 (2002).

[43] H. Luo, S. Wen, W. Shu, and D. Fan, Phys. Rev. A 81, 053826 (2010).

[44] Y. Qin, Y. Li, H. Y. He, and Q. H. Gong, Opt. Lett. 34, 2551 (2009).

[45] H. Luo, S. Wen, W. Shu, and D. Fan, Phys. Rev. A 82, 043825 (2010).

[46] K. Y. Bliokh, I. V. Shadrivov, and Y. S. Kivshar, Opt. Lett. 34, 389 (2009).

[47] M. Onoda, S. Murakami, and N. Nagaosa, Phys. Rev. E 74, 066610 (2006).

[48] W. Nasalski, Phys. Rev. E 74, 056613 (2006).

[49] Y. Gorodetski, A. Niv, V. Kleiner, and E. Hasman, Phys. Rev. Lett. 101, 043903 (2008).

[50] Y. Gorodetski, N. Shitrit, I. Bretner, V. Kleiner, and E. Hasman, Nano Lett. 9, 3016 (2009).

[51] L. T. Vuong, A. J. L. Adam, J. M. Brok, P. C. M. Planken, and H. P. Urbach, Phys. Rev. Lett. 104, 083903 (2010).

[52] O. G. Rodríguez-Herrera, D. Lara, K. Y. Bliokh, E. A. Ostrovskaya, and C. Dainty, Phys. Rev. Lett. 104, 253601 (2010).

[53] K. Y. Bliokh, Phys. Rev. Lett. 97, 043901 (2006).

[54] H. Okuda and H. Sasada, Opt. Express 14, 8393 (2006).

[55] T. A. Fadeyeva, A. F. Rubass, and A. V. Volyar, Phys. Rev. A 79, 053815 (2009).

[56] M. Merano, N. Hermosa, J. P. Woerdman, and A. Aiello, Phys. Rev. A 82, 023817 (2010).

[57] A. Bérard and H. Mohrbach, Phys. Lett. A 352, 190 (2006).

[58] P. Gosselin, A. Bérard, and H. Mohrbach, Phys. Rev. D 75, 084035 (2007). 